\title{
THE VARIABILITY OF PG1115+080
}

\author{
PAUL L. SCHECHTER \\ Massachusetts Institute of Technology
}

PG1115+080 (Weymann et al. 1980), the first quadruple lens system, remains the most promising for an optical measurement of the Hubble constant through a time delay. Factors contributing to this assessment include the well constrained geometry, the apparent magnitudes of the four components (Christian et al. 1987), the expected time delay (Narasimha \& Chitre 1992), and the position of the lensing galaxy (Kristian et al. 1993). There are nonetheless many factors which combine to render this a difficult measurement, among them the small separation of the images and the small variability amplitude and long variability time scale of the "typical" optically selected quasar (Hook et al 1994). The measurement is further complicated by the likelihood of microlensing of the individual components (Witt et al. 1995), which depends upon the degree to which the quasar is resolved and the fraction of the lens mass in microlenses.

We have monitored PG1115 with the $2.4 \mathrm{~m}$ Hiltner Telescope of the Michigan-Dartmouth-MIT Observatory for the last four seasons, with an eye toward assessing the feasibility of measuring a time delay. Our approach has been to fix the shape of the lensing galaxy and the relative positions of the four images and the galaxy with respect to each other, treating only their fluxes and their overall position as as free parameters (Schechter 1993). Using our best data the galaxy was found to be round to within $10 \%$; we have therefore taken the ellipticity to be identically zero.

We used a set of $14 \mathrm{I}$ band observations obtained over the course of two nights in 1994 April, with seeing ranging from 0.59 to 1.08 " (median of 0.74"), to obtain relative positions for the components and the shape of the galaxy. There is substantial covariance between the fluxes for components $A 1$ and $A 2$ but their sum exhibited an rms scatter, relative to star C (Vanderriest et al. 1986), of 3 millimag. The ratio of component $C$ (the third brightest and most isolated) to $A 1+A 2$ had an rms scatter of 8 millimag. The error in the mean would be considerably smaller, though at this level 

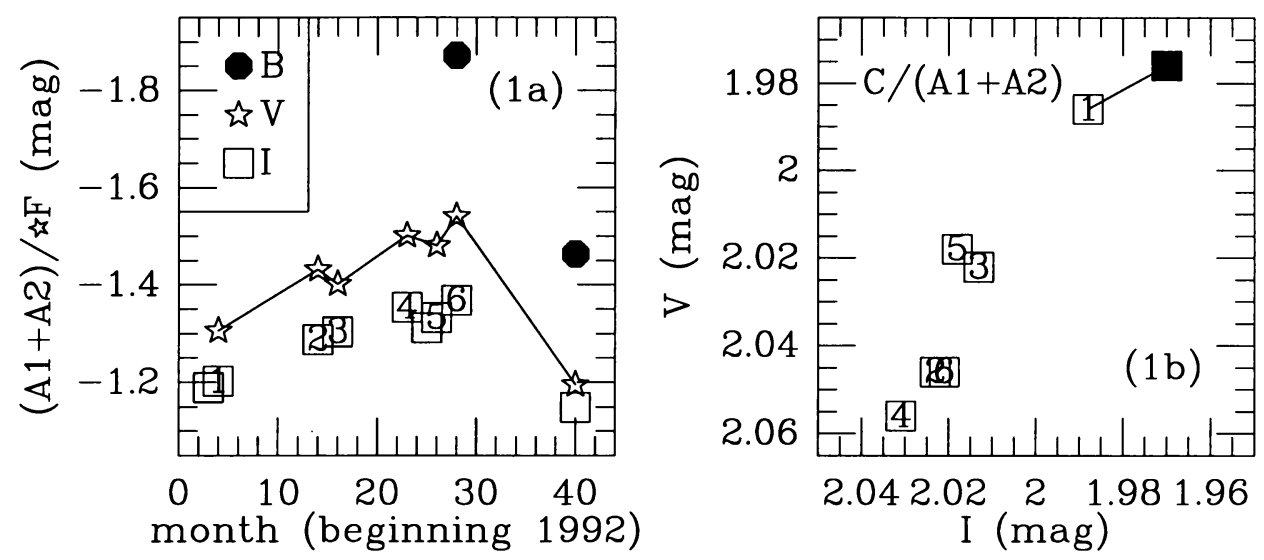

one must worry about systematic errors.

In Figure 1a we show the variability of $A 1+A 2$ relative to star $\mathrm{F}$ (Vanderriest et al. 1986) over the course of 40 months. In Figure $1 \mathrm{~b}$ we show the ratio $C / A 1+A 2$ as observed at $V$ and $I$. All of the points are derived from averages of at least 3 frames taken with the Hiltner Telescope, with the exception of 1994 April $V$ point, obtained from a single exposure, and the filled symbol, obtained at CTIO. This latter point was obtained at the same time, 1992 April, as point labeled "1". The agreement of these two, obtained with different seeing and different sampling using different instruments, supports the conclusion that the variation in $\mathrm{C} / \mathrm{A} 1+\mathrm{A} 2$ is real and not the result of systematic error. This could be due either to differential microlensing or to time delay.

\section{References}

Christian, C. A., Crabtree, D., \& Waddell, P., 1987, ApJ, 312, 45

Hook, I. M., McMahon, R. G., Boyle, B. J., \& Irwin, M. J., 1994 MNRAS, 268, 305

Kristian, J., Groth, E. J., Shaya, E. J., Schneider, D. P., \& Holtzman, J. A., et al., 1993, $\mathrm{AJ}, 106,1330$

Narasimha, D. \& Chitre, S. M., 1992, in Gravitational Lenses, eds. R. Kayser, T. Schramm \& L. Nieser, (Berlin: Springer-Verlag), 128

Schechter, P. L., 1993, in Gravitational Lenses in the Universe, eds. J. Surdej, D. FraipontCaro, E. Gosset, S. Refsdal, \& M. Remy, (Liege: Institut d'Astrophysique), 119

Vanderriest, C., Vlerick, G., Lelievre, G., Schneider, J., Sol, H., Horville, D., Renard, L. \& Servan, B., 1986, A\&A, 158, L5

Weymann, R. J., Latham, D., Angel, J. R. P., Green, R. F., Liebert, J. W., Turnshek, D. ,E., \& Tyson, J. A., 1980, Nature, 285, 641

Witt, H. J. , Mao, S., \& Schechter, P. L., 1995, ApJ, 443, 18 